\section{Acciones de capacitación para la mejora del manejo de los residuos sólidos urbanos y la protección del agua.}

\author{
Raquel de la Cruz Soriano ${ }^{1}$, Onelia Guevara Reyes, \\ Pedro Humberto Gotera Espinosa, Francisco \\ Hernández Somoza, Gretter Ledesma Santos, \\ Beatriz Presilla Andreu y Arelys García Santos \\ ${ }^{1}$ Universidad de Sancti Spíritus, Cuba \\ raquel@uniss.edu.cu
}

Training actions for the improvement of urban solid waste management and water protection.

\title{
RESUMEN
}

Recibido: $12 / 12 / 2017$

Aceptado: 20/04/2018

Publicado: 29/06/2018

Desarrollar y mantener una actitud responsable ante el cuidado y protección del medio ambiente y los recursos naturales en virtud de gestionar el desarrollo sostenible es, en Cuba, una prioridad demostrada no solo desde la concepción, organización y proyección de las políticas y proyectos que abordan estas temáticas; sino también desde los resultados concretos que exhiben. Respondiendo a este quehacer, la presente ponencia tiene como objetivo socializar la experiencia en la capacitación comunitaria para el manejo de Residuos Sólidos Urbanos y la protección del recurso agua desde la prevención en una localidad cubana. Como métodos teóricos se utilizaron: el histórico- lógico, la inducción- deducción y el análisis- síntesis; como métodos empíricos: la observación, la entrevista, la encuesta, la revisión de documentos; integrados en la Investigación-Acción-Participación y sustentados en el método dialéctico materialista. A través de estos se obtuvo un diagnóstico de las regularidades que caracterizan ambas problemáticas y se planificaron y ejecutaron un grupo de acciones dentro de las que sobresale la ejecución del proyecto: Por la salud y la cultura ambiental en comunidades de Cabaiguán. Los docentes implicados en ellas, a través de los procesos sustantivos universitarios, abordaron las temáticas con un carácter instructivo y educativo, lográndose la satisfacción en los beneficiarios.

PALABRAS CLAVE: curso de posgrado, educación ambiental, salud ambiental.

\section{ABSTRACT}

Developing and maintaining a responsible attitude towards the care and protection of the environment and natural resources by virtue of managing sustainable development is, in Cuba, a demonstrated priority not only from the conception, organization and projection of the policies and projects that address these thematic; but also from the concrete results they exhibit. Responding to this task, this paper aims to socialize the experience in community training for the management of Urban Solid Waste and the protection of water resources from prevention in a Cuban locality. Theoretical methods were used: historical-logical, induction-deduction and analysis-synthesis; as empirical methods: observation, interview, survey, document review; integrated in Research-Action-Participation and supported by the materialistic dialectical method. Through these, a diagnosis of the regularities that characterize both problems was obtained and a group of actions were planned and executed within which the project execution stands out: For health and environmental culture in communities of Cabaiguán. The teachers 
involved in them, through the substantive university processes, addressed the topics with an educational and educational character, achieving satisfaction in the beneficiaries.

KEYWORDS: environmental education, enviromental health, postgraduate course.

\section{INTRODUCCIÓN}

En la Agenda 21, planteada en la Conferencia de Naciones Unidades para el medio ambiente y el Desarrollo, convocada por la Organización de Naciones Unidas (ONU) en junio de 1992 y que tomó por sede la ciudad de Río de Janeiro, se hizo un llamado al desarrollo sostenible en todos los países. Se puso en marcha un sistema con objetivos y actividades que apuntan a minimizar los desechos y maximizar un ambiente sano mediante el reciclaje y promoción, tratamiento y disposición sostenible de aquellos.

La Agenda 2030 -como también se le conoce- ofrece inmensas oportunidades para conectar la ciencia, la sociedad y construir nuevas bases para la investigación como fuerza motriz, no solo para el desarrollo económico y la prosperidad, sino también para el desarrollo sostenible y la inclusión social (Organización de Naciones Unidas[ONU], 2015, p. 16). Dentro de ella se abordan, entre otros de igual importancia, tres temas vitales:

- Objetivo 6: Garantizar la disponibilidad de agua y su ordenación sostenible y el saneamiento para todos.

- Objetivo 12: Garantizar modalidades de consumo y producción sostenibles.

- Objetivo 13: Adoptar medidas urgentes para combatir el cambio climático y sus efectos.

Recientemente, la directora de la Organización de Naciones Unidas para la Educación, la Ciencia y la Cultura (UNESCO, por sus siglas en inglés) declaró que: el mundo está haciendo un llamado a la ciencia en la búsqueda de nuevas soluciones que sean inclusivas, para vincular las dimensiones económicas, medioambientales y sociales de la sostenibilidad (Organización de Naciones Unidas para la Educación, la Ciencia y la Cultura[UNESCO], 2017).

A su vez, Horruitinier (2006) precisa que la misión de la universidad moderna se orienta a preservar, desarrollar y promover la cultura de la humanidad, a través de sus procesos sustantivos, en plena integración con la sociedad; llegando con ella a todos los ciudadanos, con pertinencia y calidad, para contribuir así al desarrollo sostenible del país.

La educación superior cubana en correspondencia con esta necesidad, sobre bases objetivas y en plena concatenación con la política del Estado cubano expresada en el accionar de las diferentes instituciones y entidades en su desempeño, orienta su labor formativa, educativa, científica, investigativa, innovadora, y de extensión universitaria para avanzar en el logro del desarrollo sostenible.

La extensión universitaria como proceso universitario que tiene por propósito promover la cultura en la comunidad intra y extrauniversitaria para contribuir a su desarrollo cultural (González y González, 2006), es según Portero, González y Machado (2016) un proceso que integra la investigación y la docencia sin que pierdan su esencia principal; así como apoya y favorece la gestión de los mismos. Su esencia es integradora y se debe profundizar en las figuras para su mejoramiento y visualización a la luz de la globalización y los procesos que influyen en el desarrollo de la universidad actual.

Asimismo, la educación ambiental es un proceso educativo que se despliega como dimensión, perspectiva y alternativa de la educación y la pedagogía, que debe desarrollarse básicamente en la escuela por el encargo social que a esta se le confiere en la preparación de niños, adolescentes, jóvenes y adultos para la vida. La educación ambiental, desarrollada consecuentemente en la escuela y su 
proyección en la familia y la comunidad, contribuye a la formación y el desarrollo de los valores y la sensibilidad en los alumnos, en favor de la protección del medio ambiente.

La educación ambiental debe tener como base el pensamiento crítico e innovador, en cualquier tiempo y lugar, en sus expresiones formal, no formal e informal, promoviendo la transformación y la construcción de la sociedad. La educación ambiental no es neutra, sino ideológica. Es un acto político, basado en valores para la transformación social. La educación ambiental debe estimular la solidaridad, la igualdad y el respeto a los derechos humanos, valiéndose de estrategias democráticas e interacción entre las culturas (Valdés, 2002).

En este particular, resulta válido esclarecer los conceptos de educación formal; educación no formal; y educación informal. La primera se caracteriza por ser planificada y controlada por planes estables (planes de estudio), generalmente estatales o aprobados estatalmente, o jurídicamente refrendados. Es secuenciada y permanente. Tiene un público homogéneo y relativamente estable. Se refiere fundamentalmente a los procesos de escolarización de todos los niveles.

La educación no formal son procesos educativos planificados que poseen un carácter específico y diferenciado. Pueden ser o no secuenciados y controlados, y generalmente son dirigidos a públicos heterogéneos y no estables. Es el caso de las actividades extradocentes y extraescolares, las que se realizan en los parques, en las instituciones especializadas científicas y culturales, así como los procesos educativos comunitarios.

La educación informal es un proceso educativo espontáneo que resulta de la interacción del individuo con su entorno y que ocurre independientemente de la planificación institucional y familiar. Puede ser incluso, cualquier hecho fortuito que ejerza una influencia educativa (Ministerio de Ciencia, Tecnología y Medio Ambiente[CITMA], 1997).

La inadecuada gestión de los residuos supone un derroche de energía y una fuente de problemas medioambientales. Los Residuos Sólidos Urbanos (RSU) dispuestos en vertederos a cielo abierto deterioran el paisaje, emiten malos olores, son fácilmente inflamables y además presentan un grave riesgo de contaminación de las aguas tanto superficiales como subterráneas por la infiltración de los lixiviados que arrastran componentes orgánicos no estabilizados. El hombre tiene que lidiar en la actualidad con las consecuencias de la disposición de RSU a cielo abierto, cuestión aún más aguda en los países en vías de desarrollo y que está vigente en nuestro país, por lo que la toma de conciencia de las personas en la aplicación de la estrategia de las tres $\mathrm{R}$ (reducir, reciclar, reutilizar) es relevante para mitigar los efectos de la disposición de RSU.

Es una preocupante a escala global la protección del agua en un mundo donde la población y las expectativas de calidad de vida crecen. Una de las metas de la Organización de la Naciones Unidas es reducir la pobreza y asegurar el acceso de las personas al agua potable (ONU, 2003).

García (2010) refiere al planteamiento de Crosgove (2010) al expresar que hay una crisis mundial del agua, pero no es una crisis debido a la insuficiencia del recurso sino por la pobre y deficiente gestión de los recursos de agua del Planeta y afirma que toda población debe ser consciente de la importancia del agua dulce para la vida y de la necesidad de que su uso sea eficiente y responsable. En el mundo 1 100 millones de personas no disponen de agua en su casa.

Las aguas de los lagos, las presas y los ríos, así como las del manto freático son las fuentes principales de abastecimiento de agua de las colectividades humanas. Se clasifica según su uso en: aguas para uso de la población, agua para uso industrial y agua para regadío. La arcilla, los fragmentos de rocas, unido a la existencia de restos animales o vegetales en descomposición, y de microorganismos que viven en el agua son la causa de la turbidez y la coloración de esta en la naturaleza, y en muchos casos de su 
contaminación. Otras fuentes de contaminación son la industria, la actividad agropecuaria, las aguas servidas de las ciudades y comunidades.

Los porcientos de consumo en cada uso difieren en países desarrollados y países en vías de desarrollo (ONU, 2003). Igualmente existe un comportamiento promedio a escala global. En el caso de Cuba se tienen datos del comportamiento de cada uno de los consumos, se dedican recursos e infraestructura en su gestión para asegurar la satisfacción de las necesidades del recurso en todas las actividades socioeconómicas. Existe una cobertura de agua potable a la población, aunque es esencial que se conozca la dependencia de los recursos de agua con el comportamiento de las precipitaciones, variabilidad climática y la necesidad de satisfacer las demandas de la economía, la sociedad y el medio ambiente (García, 2010).

Según la UNESCO (2015) para construir un mundo con seguridad hídrica, la comunidad hidrológica mundial debe: lograr el acceso universal seguro al agua potable, saneamiento e higiene; administrar los recursos hídricos de manera sostenible a través de un enfoque de cuenca y aumentar la productividad del agua, al tiempo que se protegen los ecosistemas; administrar los recursos hídricos de manera sostenible a través de un enfoque de cuenca y aumentar la productividad del agua, al tiempo que se protegen los ecosistemas; gestionar todas las aguas residuales con el fin de proteger los recursos hídricos y los ecosistemas, aumentando el reciclaje y la reutilización y aumentar la resiliencia ante los desastres relacionados con el agua.

Esto debe ser motivación para que quienes disponen del recurso se sensibilicen con no contaminarla y ahorrarla. Los autores de la presente investigación, a partir del análisis de la literatura internacional consultada plantean la necesidad de modificar los modos de ser, de vivir, producir y consumir dicho recurso, es decir, sólo a través de profundos cambios en las relaciones hombre- naturaleza, expresado en los hábitos de las personas, la introducción de prácticas orientadas al uso eficiente de los recursos, la sostenibilidad se insertará en los valores de las comunidades, y así se transmitirá de generación a generación.

La educación ambiental se promueve desde la educación formal, no formal e informal (CITMA, 1997), e incluye dentro de sus contenidos: el ahorro de electricidad y agua; y las medidas para la protección de esta última. No obstante, a pesar de los esfuerzos realizados por diferentes gestores y actores para generalizar el conocimiento, la aplicación y la sensibilización en las entidades y la comunidad sobre el cuidado y protección del medio ambiente, el manejo de los RSU y la protección del recurso agua; existen una serie de limitaciones que han impedido avanzar eficazmente hacia la meta establecida. Entre estos aspectos se consideran:

- Insuficiente dominio o conocimiento por parte de los actores de la sociedad y de la población referidos a las normas del uso y cuidado del medio ambiente (recurso agua).

- Insuficiente planificación y divulgación de actividades que promulguen un adecuado uso y cuidado del Medio ambiente, particularmente del manejo de RSU y de la protección del recurso agua, por parte de las entidades y de la población.

- Insuficiente apoyo por parte de los factores de la comunidad para favorecer un adecuado uso y cuidado del medio ambiente.

- El tratamiento de acciones para favorecer una adecuada educación ambiental no es sistemático y en ocasiones se realiza de forma aislada.

- No se aprovecha de forma óptima el entorno cercano a la comunidad para la realización de actividades relacionadas con el tema objeto de estudio.

Es por ello que resulta necesario continuar la profundización y sistematización de diversas acciones y actividades para el logro de la comprensión y la transformación de las actitudes de todos respecto al cuidado del medio ambiente. El trabajo que se presenta tiene por objetivo socializar la experiencia en 
la capacitación comunitaria para el manejo de Residuos Sólidos Urbanos y la protección del recurso agua desde la prevención en una localidad cubana; resultado que emana de los proyectos de investigación implementados en el municipio Cabaiguán, provincia Sancti Spíritus: Proyecto de Innovación Agropecuaria Local; Proyecto para el manejo de RSU; Proyecto sociocultural del Centro Universitario Municipal «Capitán Silverio Blanco Núñez»; y proyecto Por la salud y la cultura ambiental en las comunidades de Cabaiguán.

En lo sociocultural el enfoque marxista del problema del medio ambiente se basa en el análisis de la relación hombre-naturaleza como una unidad que tiene como base la actividad humana. Tal actividad es engendrada por las necesidades objetivas, que además diseñaron nuestra especie y que se convierten en el motor de acción del pensamiento, la creación y transformación de la realidad por el hombre.

Esta relación según se proyecta de forma ideal en:

- Los conocimientos, actitudes y conductas en diversas esferas de la vida, que incluyen: las relaciones interpersonales, laborales, espirituales, políticas, científico-técnica; el cuidado de la salud; el consumo y la protección del medio ambiente.

- Valores, significados y sentidos que son resultado del proceso de interacción con la realidad a través del conocimiento, las capacidades y habilidades, los sentimientos, gustos y percepciones de los individuos.

- Intereses y necesidades de los individuos, que trazan sus proyectos de vida atendiendo a sus peculiaridades individuales, familiares, grupales de clase, y otros aspectos.

Este proyecto de vida se asienta en las peculiaridades del modo en que se oriente el desarrollo en el ámbito general, regional y local, de tal forma que las aspiraciones de los hombres, pertenecientes a grupos, clases, etnias, estarán determinadas, en última instancia, por las peculiaridades socioeconómicas en que vivan (Serrano y Fernández, 1997, p. 15).

Se consideraron los aspectos que caracterizan la dimensión ambiental en la educación formal y no formal. En la educación formal:

- Introducir la dimensión ambiental con un carácter interdisciplinario en los planes de estudio, programas, libros de texto del Sistema Nacional de Educación.

- Introducir la dimensión ambiental con un carácter interdisciplinario en los modelos del profesional, planes de estudio en sus componentes académico, laboral e investigativo, así como en los planes de ciencia y técnica del Sistema Nacional de Educación Superior.

- Promover la introducción de la dimensión ambiental en los Programas de Educación de Postgrado.

- Organizar programas de postgrado sobre medio ambiente y desarrollo y educación ambiental.

- Introducir la dimensión ambiental en la actividad extradocente, extraescolar y de extensión universitaria.

- Introducir la dimensión ambiental en los planes de superación de los profesores, incluyendo la organización de cursos básicos de educación ambiental dirigidos a todos los tipos y niveles de la educación en el país.

- Introducción de la dimensión ambiental en las actividades que vinculan la escuela con la comunidad.

- Facilitar el flujo de información sobre la problemática ambiental como elemento básico para los procesos de introducción de la dimensión ambiental. 
- Incorporar la investigación pedagógica de la educación ambiental en los planes de ciencia y técnica de la educación superior.

En la educación no formal:

- Promover la introducción de la dimensión ambiental en la actividad de los centros científicorecreativos y científico-culturales.

- Promover la introducción de la educación ambiental en la gestión de las instituciones y centros especializados vinculados a la promoción de la cultura, el deporte y la recreación.

- Desarrollar programas y proyectos educativos comunitarios, orientados a contribuir a la promoción de modos de vida sanos y relaciones de convivencia armónicas entre las personas y con su entorno natural, histórico-cultural y construido en general.

- Promover programas y proyectos comunitarios dirigidos a capacitar a las comunidades para participar de forma efectiva en la solución de sus problemas de forma autóctona y autogestionada.

- Promover la vinculación de las comunidades a actividades docentes, científicas y culturales que contribuyan a elevar su calidad de vida.

- Promover el intercambio entre líderes comunitarios de organizaciones gubernamentales, políticas y de masa y otras no gubernamentales, para contribuir a fortalecer la capacidad de participación de sus organismos y organizaciones en la solución de problemas locales.

- Promover por los organismos e instituciones gubernamentales, las organizaciones políticas y de masa y otras organizaciones no gubernamentales, proyectos comunitarios y programas educativos y divulgativos que contribuyan a la vinculación del medio ambiente con el desarrollo desde la perspectiva de su sector.

Para (Benayas y Barroso, 1995) los conceptos esenciales en la educación ambiental que intervienen la planificación de las diferentes acciones de capacitación son:

- Toma de conciencia: ayudar a los individuos y grupos sociales a sensibilizarse y tomar conciencia del entorno global y su problemática.

- Conocimientos: ayudar a los individuos y grupos sociales a comprender el entorno global, su problemática, la presencia del hombre en el entorno, la responsabilidad y el papel crítico que lo atañen.

- Actitud: ayudar a los individuos y grupos sociales a adquirir valores sociales, a interesarse por el medio ambiente, a tener una motivación fuerte para querer participar en la protección del medio ambiente y mejorarlo.

- Aptitudes: ayudar a los individuos y grupos sociales a adquirir las aptitudes necesarias para resolver problemas ambientales.

- Capacidad de evaluación: ayudar a los individuos y grupos sociales a evaluar las medidas y los programas de educación ambiental en función de los factores ecológicos, políticos, económicos, sociales, estéticos y educativos.

- Participación: ayudar a los individuos y grupos sociales a desarrollar su sentido de responsabilidad para garantizar las medidas para resolver los problemas del medio ambiente.

Al ser la educación ambiental un eje transversal común para todos los tipos de educación, es esencial definir los objetivos que esta va a desarrollar en el modelo de los diferentes niveles educacionales; precisar el sistema de conocimientos y los contenidos ambientales; y las diferentes etapas en el aprendizaje ambiental. 
En la Figura 1 se representan las etapas a lograr en la concientización de las personas desde la adquisición de conocimientos hasta el logro de una ética ambiental.

Figura 1. Etapas del aprendizaje en la educación ambiental.

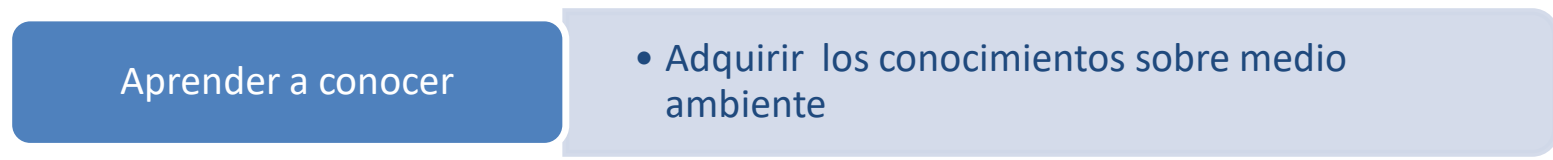

- Adquirir procedimientos que incluye aprender a seleccionar información, elegir medios y vías, formular y verificar hipótesis, relacionar datos,

Aprende a hacer establecer relaciones y llegar a conclusiones

- Participar en la gestión de los problemas ambientales

Fuente: Elaboración propia.

El proceso de enseñanza aprendizaje desarrollador constituye la vía mediatizadora esencial para la apropiación de conocimientos, habilidades, normas de relación emocional, de comportamiento y valores, legados por la humanidad, que se expresan en el contenido de enseñanza, en estrecho vínculo con el resto de las actividades docentes y extradocentes que realizan los estudiantes (Castellanos, Reinoso y García, 2000).

En (Castellanos, Reinoso y García, 2000) se precisan algunos criterios básicos de un aprendizaje desarrollador en el aprendizaje ambiental, entre ellos: promover el desarrollo integral de la personalidad del educando, es decir, activar la apropiación de conocimientos, destrezas y capacidades intelectuales en estrecha armonía con la formación de sentimientos, motivaciones, cualidades, valores, convicciones e ideales. En otras palabras, tendría que garantizar la unidad y equilibrio de lo cognitivo y lo afectivo-valorativo en el desarrollo y crecimiento personal de los aprendices. Potenciar el tránsito progresivo de la dependencia a la independencia y a la autorregulación, así como el desarrollo en el sujeto de la capacidad de conocer, controlar y transformar creadoramente su propia persona y su medio. Desarrollar la capacidad para realizar aprendizajes a lo largo de la vida, a partir del dominio de las habilidades y estrategias para aprender a aprender y de la necesidad de una autoeducación constante.

Respecto a la educación ambiental comunitaria se consideró lo abordado por Tréllez (2015, p. 7):

- No es una educación para la comunidad, ni solo en la comunidad, es una educación con la comunidad, eminentemente participativa.

- No es una educación para impartir conocimientos, es una educación para compartir conocimientos.

- No es una educación mono-disciplinaria, es una educación inter-disciplinaria, transdisciplinaria, hacia el pensamiento complejo y la construcción del saber ambiental.

- No es una educación mono-temática, es una educación integradora y multitemática.

- No es una educación teórica, es una educación teórico-práctica hacia la acción. 
- No es una educación individual, repetitiva, es una educación colectiva, hacia la construcción colectiva del conocimiento.

- No es una educación para replicar planes o programas, o seguir tendencias, es una educación para diseñar y construir futuros alternativos.

En resumen, este proceso reúne las características siguientes: altamente dinamizador; se centra en las relaciones dinámicas entre la sociedad y la naturaleza; busca conservar la naturaleza y revalorar las culturas y sus saberes; es esencialmente participativo; subraya la importancia de una relación positiva entre intelecto y emoción; combina saberes ancestrales y académicos; se orienta a la reflexión para la acción creativa; aporta a la construcción de un mundo más justo y equitativo; ofrece elementos para la construcción del saber ambiental; se sustenta en bases éticas y de valoración de las culturas; y busca mejorar la autoestima de las poblaciones y revalorar sus identidades.

Entre las limitaciones detectadas en la educación ambiental se determinó: la existencia de la educación ambiental comunitaria desde las diferentes agencias socializadoras, sin tener en cuenta el papel del Centro Universitario Municipal (CUM) como centro formador de los profesionales de la educación; insuficiente sistematización de los saberes comunitarios y la educación ambiental y el no aprovechamiento de las características socioculturales de los comunitarios para el desarrollo de una educación ambiental comunitaria desde una perspectiva del desarrollo sostenible local.

Por otra parte, las potencialidades con que cuenta el CUM para la planificación, ejecución y control de acciones son las siguientes:

- Se dispone de una estrategia ambiental de la institución.

- Como institución universitaria formadora de profesionales desempeña un rol importante como socializador en la comunidad.

- Posibilidad de liderar y organizar actividades formativas hacia una educación ambiental a la comunidad a través de la extensión universitaria.

- Posibilidad de agrupar las diferentes agencias socializadoras y promover, la participación, la reflexión y el análisis conjunto de las necesidades educativas, culturales entre otras y desarrollar una educación ambiental comunitaria.

- El carácter activo de los sujetos que intervienen en el proceso de formación de educación ambiental con carácter transformador.

\section{MATERIALES Y MÉTODOS}

Como métodos teóricos se utilizaron: el análisis y síntesis para el estudio de la problemática en el manejo de los residuos sólidos y en la protección y uso racional del agua en la localidad; así como para el proceso de enseñanza- aprendizaje en las diferentes actividades docentes, con el fin de seleccionar y sintetizar las que mayor connotación, relevancia e importancia tuvieron en la proyección de las acciones; inducción-deducción, en aras de establecer generalizaciones en relación con los resultados científicos de la investigación, a partir del análisis particular de los criterios de disímiles autores y de la teoría científica e histórico-lógico, al evaluar las dos temáticas en su desarrollo a partir de sus antecedentes, situación problemática, sus causas y efectos en los diferentes espacios, y las posteriores acciones propuestas y ejecutadas.

Como métodos empíricos: la observación, la entrevista, la encuesta, y la revisión de documentos; integrados en la investigación-acción-participación y sustentados en el método dialéctico materialista. Las operaciones que conforman las acciones van a depender de las condiciones concretas en que se realizan y de los medios e instrumentos que el individuo tenga a su disposición para llevarlas a cabo. Puede decirse que la acción constituye el proceso subordinado a una representación del resultado a 
alcanzar, o sea, a una meta u objetivo conscientemente planteado mientras que la operación se define como las formas y métodos por cuyo intermedio se realiza aquella.

Específicamente en la presente experiencia, la propuesta de acciones se diseñó a partir de los rasgos generales determinados para estas por un colectivo de autores de la Universidad de Ciencias Pedagógicas «Félix Varela», los que las caracterizan desde un enfoque sistémico en el que predominan las relaciones de coordinación, estructuradas por etapas en las que a su vez intervienen acciones de orientación, ejecución y control, con caracteres dialéctico, contextual, personalizado, dinámico, objetivo, operativo, formativo y sistemático.

La observación permitió constatar la problemática en cuanto al manejo de los RSU y la no aplicación correcta de medidas de ahorro y protección del agua. La entrevista, facilitó identificar la necesidad de capacitación en la temática a los diferentes sectores y en la comunidad, tales como comunidades rurales, la Cátedra Universitaria del Adulto Mayor, la agricultura, instituciones de educación, etc. La revisión de documentos posibilitó consultar la bibliografía necesaria para definir conceptos; conocer los procedimientos correctos acorde con la pedagogía para la educación ambiental; los contenidos específicos de las temática a abordar; entre otros aspectos de rigor que garantizan la preparación de las actividades docentes, la realización de las investigaciones científicas, su comunicación en forma de artículos científicos y ponencias, así como la proyección de las acciones socioculturales y de recreación sana y de contenido político y patriótico en correspondencia con los valores de nuestra sociedad socialista.

La consulta a profesionales se realizó para precisar aspectos de rigor técnico tanto de carácter teórico como práctico en historia local; elementos relevantes de personalidades significativas de nuestra historia; datos técnicos en los consumos de agua en la agricultura; y formas de manejo de RSU. A raíz, se planificaron diferentes acciones de capacitación teniendo en cuenta su carácter dinámico, flexible, integrador, dialéctico, participativo, con la retroalimentación y participación de los implicados en las mismas.

Los recursos tecnológicos permitieron el empleo de presentaciones en diapositivas, postales digitales y obras audiovisuales y musicales para fomentar la sensibilización de la problemática del agua. Se consideraron los principios filosóficos marxistas-leninistas fundamentales: análisis multilateral, el historicismo y el análisis sistémico. La encuesta se utilizó para medir el nivel de satisfacción de los participantes con las actividades realizadas.

\section{Acciones ejecutadas.}

a) Acciones desde el proceso de formación de pregrado:

- Educación ambiental desde la Práctica Laboral e Investigativa para estudiantes de carreras pedagógicas.

- Implementación de la Estrategia ambiental durante el proceso de formación a través de la clase, en las carreras que se imparten en el CUM, curso escolar 2016-2017.

b) Formación de posgrado:

- Impartición de cursos de posgrado sobre ahorro y uso racional del agua en Maestrías.

- Impartición de cursos de capacitación: El agua un recurso vital. Cómo podemos ahorrarla.

- Impartición de conferencias especializadas a recursos humanos del sector agropecuario y estudiantes en formación.

c) Acciones desde el proceso de ciencia, tecnología e innovación:

- Elaboración de ponencias para fórum estudiantil de secundaria básica.

- Atención a sociedades científicas en el preuniversitario de la localidad.

- Elaboración y ejecución de proyectos. 
- Proyecto Por la salud y cultura ambiental en comunidades de Cabaiguán (actividades de capacitación; intervención comunitaria; elaboración de software educativos; charlas educativas; programas radiales, entre otros).

- Participación en eventos científicos de carácter local, provincial, nacional e internacional.

- Publicación de artículos científicos.

- Obtención de un premio de Innovación tecnológica a nivel provincial.

d) Acciones desde el proceso de extensión universitaria:

- Realización de charlas comunitarias. Participan: amas de casa, adultos mayores, promotora cultural, profesores del Instituto Nacional de Deporte y Recreación, bibliotecaria, pioneros de enseñanza primaria, maestros, cuadros.

- Impartición de cursos de capacitación a la Cátedra Universitaria del Adulto Mayor (CUAM). Participan: miembros de la CUAM, vecinos, Federación de Mujeres Cubanas, profesores del CUM.

- Acciones de higienización y reforestación en zonas urbana (ESBU Juan Santander) en conmemoración por el Día mundial del Medio ambiente (año 2016 y año 2017). Participan: estudiantes y profesores de secundaria básica, vecinos, organizaciones del municipio, gobierno.

- Lanzamiento y premiación de concursos dirigidos a pioneros de enseñanza primaria por motivo del Día mundial del Agua y del Día mundial del Medio ambiente (vínculo con lo extracurricular en la educación primaria).

- Elaboración y entrega de plegables con contenido orientador sobre la solución de problemas ambientales y la prevención. (Acción comunicativa). Realización de programas radiales. (Acción comunicativa).

- Presentación y reflexión de audiovisuales para la sensibilización. (Acción comunicativa).

- Celebración de efemérides conmemorativas del medio ambiente con la integración de la escuela, la comunidad y las organizaciones de masa.

\section{RESULTADOS Y DISCUSIÓN}

Entre los resultados más relevantes obtenidos se encuentra:

- Curso de capacitación a la CUAM sobre ahorro y protección del agua. 30 participantes (2014, 2015, 2016 y 2017).

- Realización del taller Tus iniciativas para promover el ahorro del agua. 15 participantes en uno de los patios comunitarios.

- Charla educativa sobre la importancia del agua para los seres humanos. 22 participantes de la CUAM y uno de los patios comunitarios $(2016,2017)$.

- Curso de capacitación: Manejo adecuado de residuos sólidos urbanos. Participación de 30 adultos mayores de la CUAM (2016 y 2017).

- Charlas educativas en consejos populares de zonas rurales.

- Charlas educativas en los Patios Comunitarios.

- Participación de 120 pioneros en los concursos convocados y premiación de los mejores resultados.

- Impartición de un curso pre-evento en el Evento Provincial Universidad 2018.

En la Figura 2 se totalizan los participantes en la capacitación en ambas temáticas. 
Figura 2. Total de participantes en diferentes acciones de capacitación.

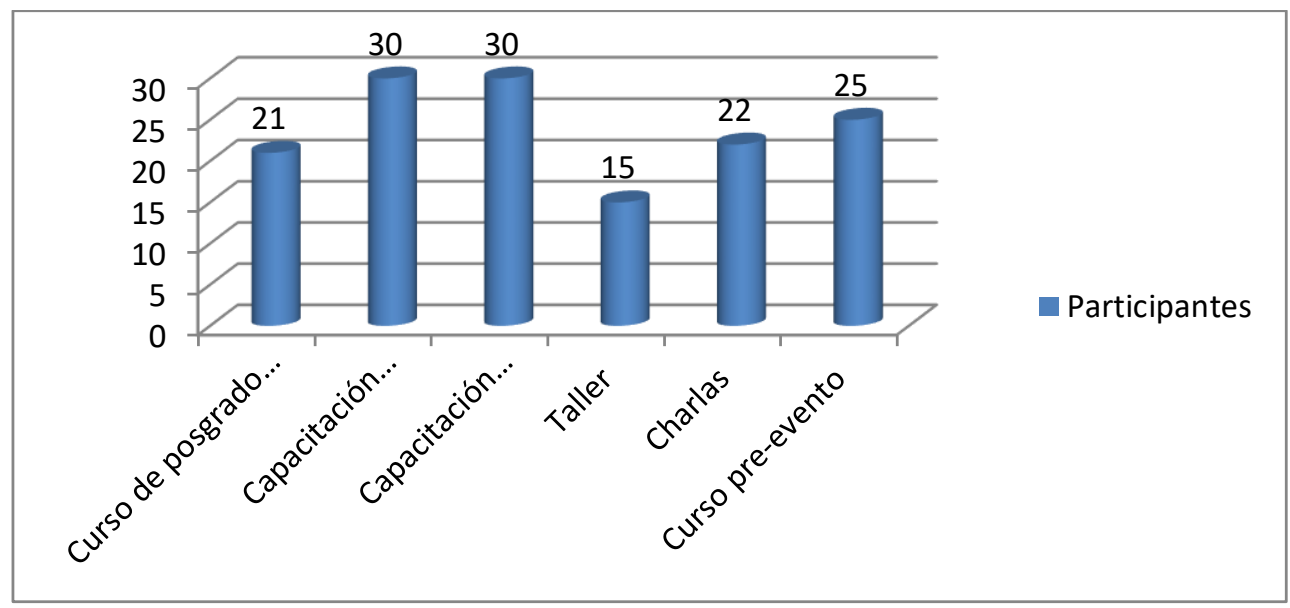

Fuente: Elaboración propia.

\section{RESULTADOS FINALES}

Se logró como resultado contribuir a la educación ambiental de los participantes en cuanto al incremento de los conocimientos sobre las prácticas para el reciclaje, la reutilización y la reducción del consumo en la disposición de los RSU y sobre el ahorro de agua y su protección. La socialización de procedimientos y actividades para lograr implementar prácticas que mitiguen los efectos negativos sobre el medio ambiente; por ejemplo: uso de plástico para elaborar adornos florales, uso de recortes de tejido para elaborar medios y manualidades de uso doméstico, reutilizar bolsas de nylon en la plantación de viveros, cómo ahorrar el agua en el hogar en los diferentes usos. Se han utilizado plegables, ponencias y un folleto.

La evaluación de la satisfacción con las actividades comunitarias como proceso de retroalimentación, a través de la encuesta aplicada a una muestra de 35 participantes en las diferentes actividades ejecutadas. Se resumió lo siguiente:

El 97\% de la muestra ha participado en las acciones de capacitación y socioculturales realizadas por el Centro Universitario Municipal de Cabaiguán. El comportamiento de la participación en diferentes tipologías se comporta de la forma mostrada en la Figura 3.

Figura 3. Formas de participación de los encuestados.

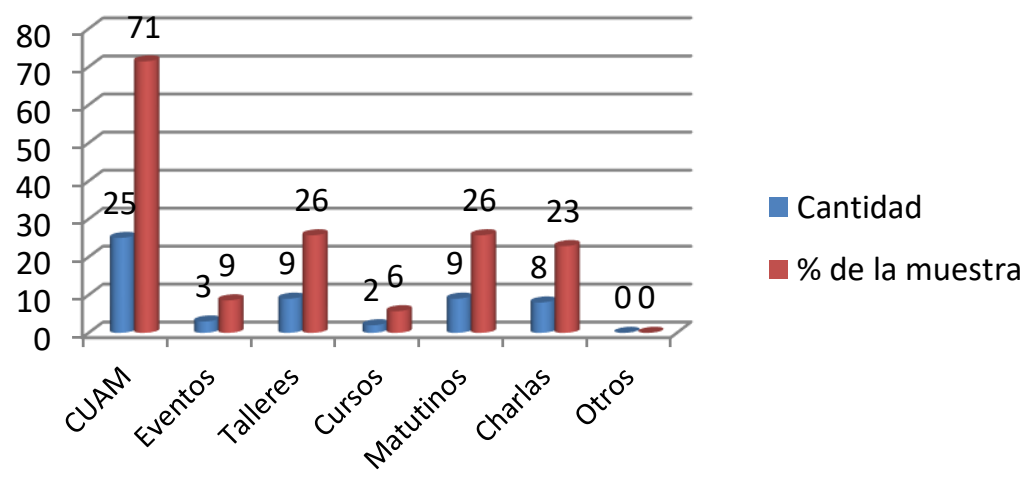

Fuente: Elaboración propia. 
El 94\% refiere que la participación le ha permitido adquirir nuevos conocimientos sobre la protección del medio ambiente; el $97 \%$ que le ha aportado ideas y experiencias para mejorar la protección del medio ambiente y los recursos naturales en su casa y en la comunidad; y el $97 \%$ ha reconocido que se han sensibilizado con la temática, convirtiéndose en multiplicadores y transmisores de los conocimientos adquiridos para cuidar el medio ambiente y los recursos naturales.

La calidad de las actividades se expresó en un $71 \%$ de excelente y un $29 \%$ de bien.

Las temáticas muestran un comportamiento según la Figura 4.

Figura 4. Comportamiento de las temáticas para otras actividades.

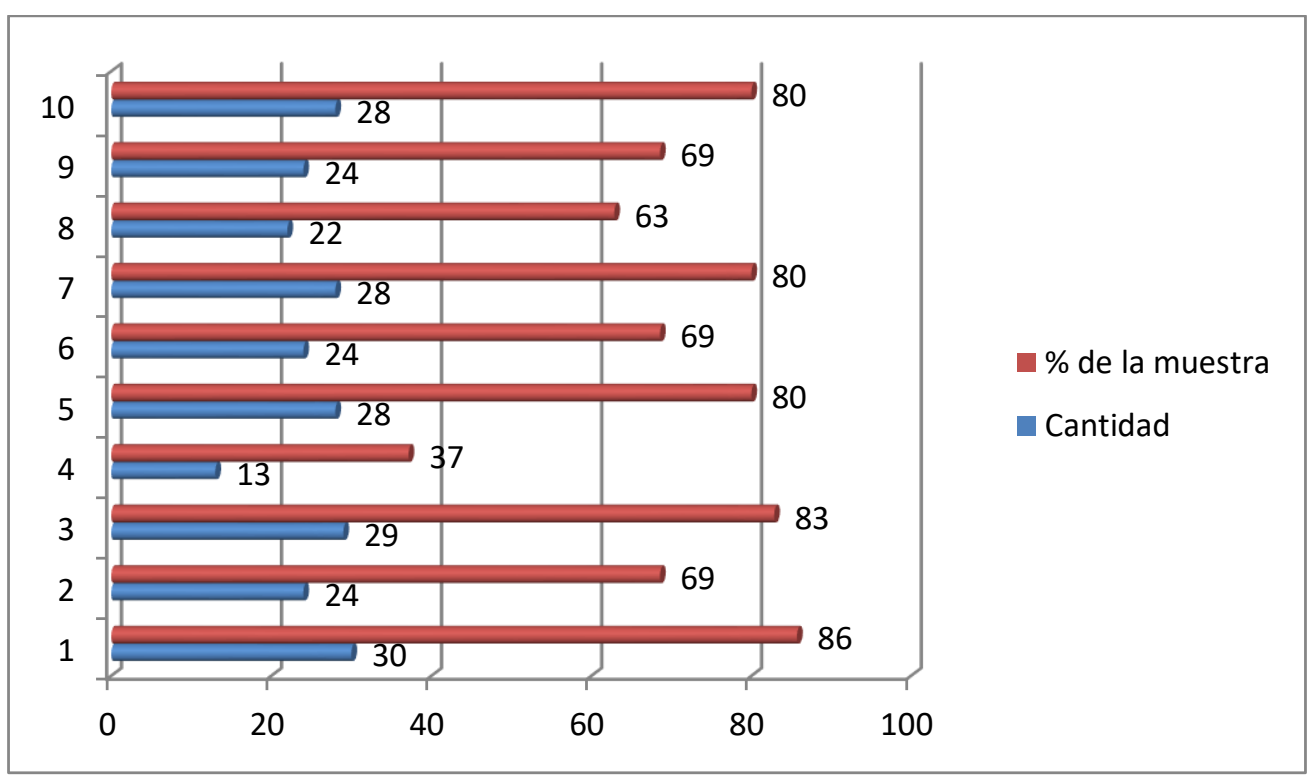

Nota. 1) Reducir el consumo de electricidad y agua. 2) Reducir el consumo de electricidad y agua. 3) Reducir el consumo de electricidad y agua. 4) Reutilizar. 5) Reciclar. 6) Reforestar. 7) Prácticas para la protección de la fauna. 8) Prácticas para la protección de las aguas. 9) Prácticas para la protección del suelo. 10) Divulgación ambiental. 11) Respetar la legislación ambiental. 12) Prácticas para prevenir los riegos asociados a los desastres naturales. Fuente: Elaboración propia.

Otras temáticas que solicitan sean abordadas: la salud en el adulto mayor; la prevención de las ITS y el VIH SIDA; el alcoholismo; temas sobre jardinería y plantas ornamentales; visitar lugares históricos.

Sugieren para mejorar la calidad: realizar más audiovisuales, historias de vida de personas que hayan superado el alcoholismo, convocar a otros centros de trabajo de la comunidad a que participen, incrementar el número de actividades y que se realicen con mayor sistematicidad.

\section{CONCLUSIONES}

Se logró abordar desde los procesos sustantivos, con contenidos instructivos y educativos, las dos temáticas referidas en diferentes actividades docentes, investigativas y extensionistas tanto en la comunidad universitaria como extramuros. Igualmente se premiaron los concursos convocados para pioneros de primaria; se logró la participación en eventos de sociedades científicas de preuniversitario; la presentación de resultados en eventos científicos; la publicación de artículos; la obtención de un premio de innovación tecnológica y la impartición de un curso pre-evento. 
Se fomentó la sensibilización de la población en la necesidad del cuidado del entorno; se materializa la labor educativa con un contenido teórico-práctico, vinculado a la celebración de fechas históricas significativas a nivel internacional, nacional y local, con énfasis en los hechos históricos y las personalidades relevantes de nuestra nación; y se logra un nivel de satisfacción expresado por los encuestados, quienes evalúan las actividades realizadas de excelente y manifiestan haber transmitido los conocimientos adquiridos a la comunidad; y consideran los contenidos ambientales necesarios a tratarse en nuevas actividades.

\section{REFERENCIAS BIBLIOGRÁFICAS}

Benayas, J. y Barroso, C. (1995). Conceptos y fundamentos de la Educación Ambiental. Historia y antecedentes. Monografías Master EA, Instituto de Investigaciones Pedagógicas de Málaga.

Castellanos, D., Reinoso, C. y García, C. (2000). Para promover un Aprendizaje Desarrollador. Instituto Superior Pedagógico «Enrique José Varona». https://n9.cl/fguj

García, F. (2010). Sobre los recursos hídricos internacionales y nacionales. Consejo Nacional de Cuencas Hidráulicas.

González, M. y González, G.R. (2005). Extensión universitaria: Modelo teórico y de gestión para la educación superior cubana [Monografía]. https://n9.cl/c3ln

Horruitinier, P. (2006). La universidad cubana: el modelo de formación. Editorial Félix Varela.

Ministerio de Ciencia Tecnología y Medio Ambiente. (1997). Estrategia Nacional de Educación Ambiental 2016-2020.

Organización de las Naciones Unidas para la Educación, la Ciencia y la Cultura. (2015). Agua, sociedad y cooperación: 50 años de los programas sobre agua para el desarrollo sostenible de la UNESCO. https://unesdoc.unesco.org/ark:/48223/pf0000235029

Organización de las Naciones Unidas para la Educación, la Ciencia y la Cultura. (2017). Mobilising UNESCO Chairs in Natural Sciences for Policy Action towards the 2030 Agenda. Organización de las Naciones Unidas para la Educación, la Ciencia y la Cultura. https://n9.cl/kiam

Organización de Naciones Unidas (2003). Agua para todos, agua para la vida: informe de las Naciones Unidas sobre el desarrollo de los recursos hídricos. https://unesdoc.unesco.org/ark:/48223/pf0000129556 spa

Organización de Naciones Unidas (2015). Transformar nuestro mundo: la Agenda 2030 para el Desarrollo Sostenible. https://unctad.org/meetings/es/SessionalDocuments/ares70d1 es.pdf

Portero, A., González, M. y Machado, J.R. (2016). La extensión universitaria como proceso sustantivo integrador. Organización necesaria. Referencia Pedagógica. 4(2). 193 - 206. https://n9.cl/7ncq

Serrano, H. y Fernández, A. (Coord.). (1997). Introducción al estudio del Medio ambiente. Editorial Academia.

Tréllez, E. (2015). Educación ambiental comunitaria en América Latina. PNUMA/ROLAC. https://n9.cl/xda9 
Valdés, O. (2002). ¿Cómo ha evolucionado el concepto de educación ambiental: qué resultados y limitaciones se han obtenido y cuáles son las proyecciones hacia el tercer milenio? [CD-ROM]. La Habana: Empresa de Desarrollo de Software de Calidad. 